\title{
An extreme case of platypnoea-orthodeoxia syndrome
}

\author{
Authors: Kevin O'Gallagher, ${ }^{A}$ Evelyn Chou, ${ }^{B}$ Swarna Jeyabraba, ${ }^{B}$ Aish Sinha, ${ }^{C}$ Daniel Robb ${ }^{D}$ and Jonathan Byrne ${ }^{E}$
}

An 80-year-old female presented with progressive breathlessness, worse on sitting or standing and relieved by lying flat. Subsequent investigations identified a patent foramen ovale (PFO) with right-to-left flow across the interatrial septum (IAS). A diagnosis of platypnoea orthodeoxia syndrome secondary to inter-atrial shunting was made. Technical features precluded a percutaneous PFO closure so an open surgical repair was performed with complete resolution of symptoms. We discuss the pathophysiology and management of platypnoea orthodeoxia syndrome.

KEYWORDS: Platypnoea, orthodeoxia, echocardiography

\section{Case presentation}

An 80-year-old female patient presented with an 8-week history of progressive breathlessness. Initially, she was symptomatic when seated or standing, but not when lying flat. Her symptoms progressed such that she was rendered bedbound.

Additionally, the patient had been experiencing back pain over the previous month, temporally associated with the onset of her breathlessness. Her height had reduced from 154 to $146 \mathrm{~cm}$.

Her past medical history included osteoporosis - with associated fractures of T8 and L1 vertebrae - and essential hypertension.

On examination, she was found to have a heart rate of $105 \mathrm{bpm}$, respiratory rate of 21 breaths per minute and oxygen saturations of $87 \%$ (on $8 \mathrm{~L}_{2}$ ) while seated, $97 \%$ (on $8 \mathrm{~L} \mathrm{O}_{2}$ ) while lying flat. A pan-systolic murmur was audible. Chest examination was unremarkable. She was noted to have a marked kyphosis.

\section{Diagnosis}

Considered in isolation, the patient's presenting symptom of breathlessness has a wide differential diagnosis, including

Authors: ${ }^{\text {A }}$ specialty registrar in interventional cardiology, King's College Hospital NHS Foundation Trust, London, UK; B ${ }^{\text {medical }}$ student, King's College School of Medical Education, King's College London, London, UK; ' C senior house officer, King's College Hospital NHS Foundation Trust, London, UK; ${ }^{\mathrm{D}}$ consultant in cardiothoracic surgery, King's College Hospital NHS Foundation Trust, London, UK; ${ }^{E}$ Consultant cardiologist, King's College Hospital NHS Foundation Trust, London, UK common cardiac and respiratory causes. However, the fact that the patient's breathlessness was worse on sitting/standing and relieved by lying flat is a striking feature and was the opposite of what would be expected in congestive cardiac failure. The working diagnosis was therefore the rare cardiac condition platypnoea-orthodeoxia syndrome (POS).

\section{Initial management}

Management of POS involves identifying and addressing the underlying cause, which is usually a defect in the interatrial septum (IAS) that allows shunting of blood. Because of the severity of the patient's symptoms, initial management involved hospitalisation while further investigations were carried out.

\section{Case progression}

Transthoracic echocardiography demonstrated normal biventricular function. A mobile interatrial septum was noted. An interatrial communication was confirmed on bubble echocardiography.

A transoesophageal echocardiogram, performed in both the supine and seated positions, identified a defect in the IAS with right-to-left shunting. The shunt was present with the patient supine, increasing in severity when the patient was upright.

A right and left cardiac catheterisation was performed. Right heart pressures were normal, with the patient both supine and seated. Differential oxygen saturations, measured with the patient both supine and sitting, allowed quantification of the interatrial shunt. The right-to-left shunt ratio yielded values of 1.6:1 when lying flat, increasing to 1.9:1 when seated. Coronary angiography demonstrated minor atheroma only.

Computed tomography (CT) thorax demonstrated no evidence of aortic aneurysmal disease. No lung pathology was noted. CT pulmonary angiography failed to demonstrate any pulmonary embolic disease or abnormality in pulmonary vasculature. Incidental note was made of a new T12 compression fracture, in addition to the known vertebral fractures. Cardiac magnetic resonance imaging showed a small right atrium.

Liver function tests were normal.

On the basis of these investigations, a confirmed diagnosis was made of POS secondary to interatrial shunting through a patent foramen ovale (PFO).

Technical considerations meant that the patient was unsuitable for a percutaneous PFO closure (lack of an anterior rim to the interatrial defect) so the patient underwent an 

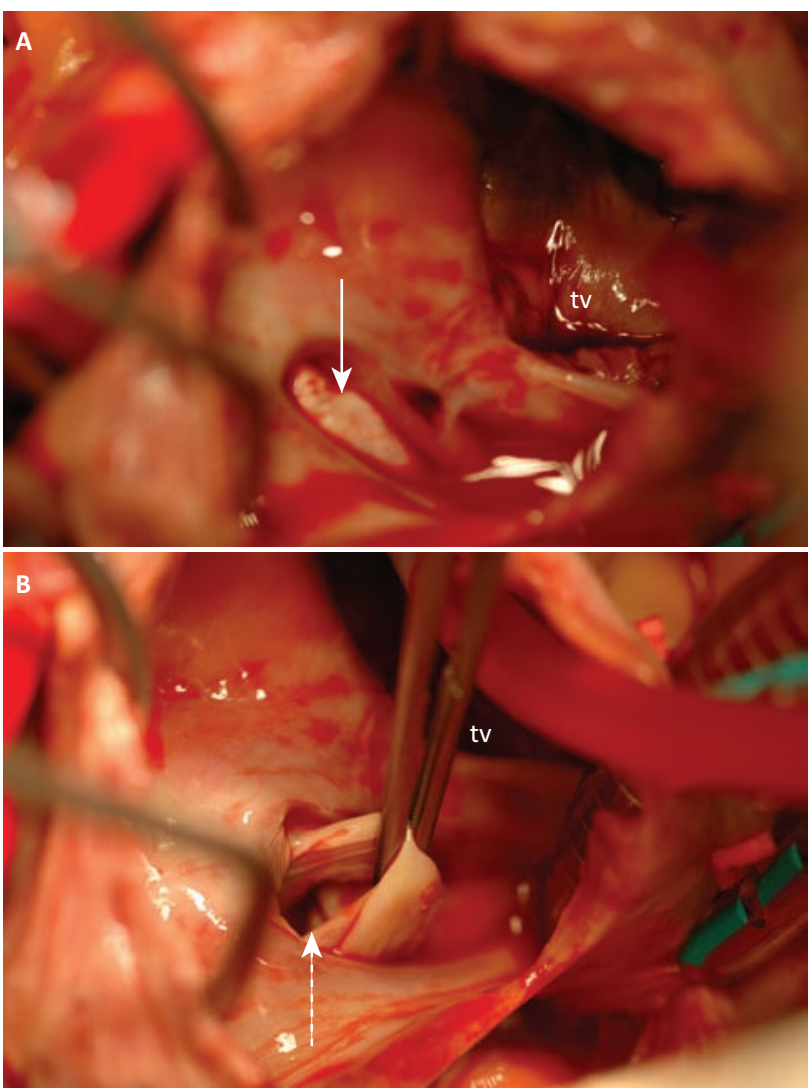

Fig 1. Intra-operative view of right atrial floor and interatrial septum. A - Flap of pale septal tissue overlying the interatrial defect (solid arrow). B - Forceps retracting septal tissue to reveal patent foramen ovale (dotted arrow). $\mathrm{tv}=$ tricuspid valve.

open surgical repair. Direct visual assessment confirmed the presence of a PFO (Fig 1), which was closed. Intra-operative transoesophageal echocardiography confirmed resolution of the interatrial shunt.

The patient was discharged with complete symptomatic relief and return of normal oxygen saturation levels.

\section{Discussion}

POS describes the combination of orthostatic breathlessness with evidence of orthostatic arterial desaturation. As in this case, POS is most commonly described in patients with right-to-left interatrial shunting. ${ }^{1}$ Other recognised causes include pulmonary arteriovenous malformation, ${ }^{2}$ large ventilation:perfusion mismatch of any cause, ${ }^{3}$ and hepatopulmonary syndrome. ${ }^{4}$

When one considers the total number of reported cases of POS $(188)^{2}$ compared with the prevalence of PFO (approximately $10 \%)$ in the adult population, it is apparent that the presence of an interatrial defect is not in itself sufficient to bring about POS. ${ }^{5}$ There must also exist a functional component to facilitate shunting.

In this case, in addition to the anatomical components (the PFO and an abnormally small right atrium), this patient had two functional components. Firstly, intra-operatively she was found to have a dilated aortic root, which has the effect of effacing the IAS. Secondly, she had an acute progression of her kyphosis due to vertebral collapse. These functional components change the anatomical relationship in the right atrium resulting in preferential blood flow directed toward the IAS when upright.

As in this case, right-to-left interatrial shunting in POS classically occurs in the setting of normal right heart pressures, supporting the hypothesis that it is a flow-mediated, rather than a pressure-mediated phenomenon.

Importantly, although this patient had all the hallmarks of POS, by the time of presentation to hospital her condition had progressed to the stage that it was not strictly-speaking platypnoea orthodeoxia because neither her breathlessness nor hypoxia resolved completely on lying flat. This patient therefore had a particularly severe form of POS that extends outside the boundaries of the current disease definitions.

\section{Key learning points}

$>$ Although there is a high prevalence of $\mathrm{PFO}$ in the population, POS remains rare.

> It is important to fully characterise the nature of a patient's breathlessness when considering the differential diagnosis.

$>$ The presence of platypnoea in a patient history clearly suggests the need for specialist cardiology review

\section{Conflicts of interest}

The authors declare no conflicts of interest.

\section{Acknowledgements}

Written informed consent was obtained from the patient to publish the clinical details and images in this article.

\section{References}

1 Rodrigues P, Palma P, Sousa-Pereira L. Platypnea-orthodeoxia in review: defining a new disease? Cardiology 2012;123:15-23.

2 Kumar N, Kraemer RR, Murthy RK et al. Platypnea-orthodeoxia syndrome as a presentation of hereditary hemorrhagic telangiectasia. Circulation 2012;126:2645-7.

3 Yamakawa $\mathrm{H}$, Yoshida M, Baba Y et al. Reversible platypneaorthodeoxia syndrome induced by rapidly progressive interstitial pneumonia in a patient with polymyositis. Respiriol Case Rep 2014;2:91-4.

4 Poterucha JJ, Krowka MJ, Disckson ER et al. Failure of hepatopulmonary syndrome to resolve after liver transplantation and successful treatment with embolotherapy. Hepatology 1995;21:96-100.

5 Cheng TO. Platypnea-orthodeoxia syndrome: etiology, differential diagnosis, and management. Cathet Cardiovasc Interv 1999;47:64-6.

Address for correspondence: Dr K O'Gallagher, Department of Cardiology, King's College Hospital, Denmark Hill, London SE5 9RS, UK.

Email: drkogallagher@gmail.com 